\title{
Respiratory intensive care in Europe: lessons for the UK
}

\author{
M W Elliott, S V Baudouin
}

In this issue of Thorax Nava et al review the provision of respiratory intensive and high dependency care in Europe. ${ }^{1}$ Medically orientated high dependency units (HDUs) are rare in the UK and in a recent survey only $26 \%$ of 190 general hospitals with an intensive care unit (ICU) had an $\mathrm{HDU}^{2}$; the proportion of beds allocated for medical patients was not stated. With increasing pressure on intensive care beds and the development of non-invasive ventilation, it is timely to consider the provision of a clinical area intermediate between intensive care and a general medical ward. Common sense suggests that, if the choice is between an ICU - with one nurse to each patient and a high level of monitoring - and a general ward-with a much lower nurse:patient ratio and little or no monitoring equipment - patients will either need to remain in the ICU longer than is necessary or be discharged to the ward earlier than is ideal.

However, we live in an era of evidence based medicine and business cases. A recent Medical Research Council/ Department of Health Working Party paper ${ }^{3}$ highlighted the fact that little hard evidence exists, in the form of clinical controlled trials, to prove that HDU (or ICU) care is effective or even cost effective. This makes it difficult to argue for increasing provision of these facilities or to decide on the best way to organise this type of care. There are some persuasive financial and organisational arguments in favour of the establishment of HDUs. Significant financial savings could be made by caring for some proportion of the current ICU population in an HDU. A number of studies indicate that a significant proportion (23-33\%) of ICU beds are occupied by patients who could be cared for on HDUs. ${ }^{4-6}$ The saving of ICU bed days should, in theory, translate into significant cost savings. However, reliable quantitative information is rare in the field of ICU costings, due in part to the complexity of indirect costing. Some progress has been made and one study found that an ICU bed day in a teaching hospital cost $£ 1149$ in 1991 compared with $£ 437$ for an HDU bed day. ${ }^{7}$ The fact that up to one third of all patients in ICUs could be cared for in an area with only one third of current ICU costs would seem attractive when persuading purchasers of the need for HDUs. However, the predicted cost savings may not occur due to the phenomenon of "unmet need" in the system. In the above study, for example, the majority of HDU admissions had medical problems at presentation and would probably have been cared for on general medical wards if the HDU did not exist. Rather than cost saving, therefore, the creation of an HDU may drive up total hospital costs. This is borne out by the fact that, over a three year period, HDU costs rose by $50 \% .^{7}$ Identifying funds for a new HDU is therefore likely to be difficult. Purchasers will be wary of arguments involving ICU cost savings as they know that the low UK provision of ICUs is acting as an effective rationing system for this expensive resource. They will recognise that ICU beds that are released by movement to an HDU will soon be filled by other patients.

Organisational and efficiency arguments for an HDU are more likely to be successful. Reasons for concentrating all the ill patients in a hospital within one area include the diminishing number of experienced trainees and nurses available on general wards, the comparative rarity of severe physiological disturbance in unselected "acute" medical admissions, and the effective concentration of expensive equipment and technology. Another factor that may drive the development of HDUs is the move towards regionalisation of intensive care facilities which is already occurring in the paediatric field. In this model the more complex intensive care patients with multi-organ involvement would be moved to regional units. The remaining patients could then be managed in an HDU with some short term intensive care capacity. In addition, the emergence of non-invasive ventilation, usually provided by respiratory physicians, for acute on chronic respiratory failure is a major stimulus for the development of some form of higher dependency facility outside the ICU. Three randomised controlled trials ${ }^{8-10}$ and a number of uncontrolled studies have suggested a potential benefit for noninvasive ventilation in acute exacerbations of COPD. Although all can be criticised and further studies are needed, there is a growing momentum for provision of this service in most acute hospitals. Non-invasive ventilation is felt to be a time consuming procedure for nurses ${ }^{11}$ and it is noteworthy that two of the trials ${ }^{9}{ }^{10}$ showing benefit were performed on ICUs; one other study ${ }^{12}$ performed on a general ward did not show any advantage though the patients were only mildly acidotic and the numbers small. If non-invasive ventilation is to be effective it is likely that a higher level of input than that available on most general wards will be needed.

Clear operational policies need to be developed if proposed HDUs are to run efficiently. ${ }^{13}$ Admission and discharge from an HDU will require careful control. In simple terms admission to an HDU should be judged on a combination of illness severity, the ability to treat the condition effectively, and the prospect of useful patient recovery. Guidelines are needed on patient selection for intensive care rather than high dependency care. Patients who are unable to breathe spontaneously should be admitted to intensive care units as should most who develop two or more organ system failures. The likelihood of the latter group needing full mechanical ventilatory support are very high. Modifications of existing intensive care severity scoring systems could provide a useful screening tool for ward use and should be developed to aid selection of cases for admission to HDUs. ${ }^{6}$ A clear policy on patient discharge from high dependency is also required.

It is suggested that the ratio of nurses to patients for an HDU be one nurse to two patients ${ }^{3}$ although there are no hard data to support this recommendation. Experience from Europe suggests that a lower staff:patient ratio may be acceptable on a dedicated respiratory unit. A high dependency area on a respiratory ward may be an alternative to a dedicated HDU, allowing the more efficient deployment of nursing staff and therefore a lower nurse:patient ratio. However, such areas must be adequately resourced both in terms of staffing, with an increase in the general ward staff complement (though further work is needed to determine what staff are needed), and equipment. It is not appropriate simply to relabel existing resources. It is important that nursing and medical staff working on HDUs receive adequate training, particularly in mechanical ventilation and the problems associated with the non-invasive approach. A period of attachment to an established HDU 
and ICU would be useful. Most UK trained respiratory physicians do not have the necessary skills with ventilators and monitoring to run a respiratory intensive care unit of the type discussed by Nava et al. ${ }^{1}$ However, non-invasive ventilation currently falls largely within the domain of respiratory physicians and experience of non-invasive ventilation should be part of the training of all specialist registrars in respiratory medicine.

\section{Conclusions}

In much of Europe the intensive care of acute respiratory failure is the province of respiratory physicians, whereas in the UK it largely falls to anaesthetists. It is clearly not feasible to move to the European model as outlined by Nava et al without a considerable change in the training of UK respiratory physicians. Two approaches to the development in the UK of medically orientated HDUs are possible. In one, respiratory physicians on their own could provide non-invasive modes of ventilation and a more appropriate level of care for patients with acute severe respiratory disease in an area within or adjacent to a home ward. An alternative involves a hard examination of the use of existing hospital acute service areas including coronary care. The immediate care of all ill patients in many primary centres may then be better met by a flexible HDU/CCU with some short term intensive care capability. The care of this group of patients would then involve a team drawn from anaesthesia, cardiology, and respiratory medicine. A few hospitals have adopted this approach and the advantages and possible disadvantages should be further examined. In either case the emergence of non-invasive positive pressure ventilation and the current interest in HDUs gives respiratory medicine a unique opportunity to broaden its horizons. It would be a pity if this was missed.

$\mathrm{M}$ W ELLIOTT

Consultant Respiratory Physician,

St Fames's University Hospital,

Beckett Street,

Leeds LS9 7TF, UK

Senior Lecturer in Anaesthesia and Intensive Care and Honorary Consultant Physician,

Royal Victoria Infirmary,

Nerwcastle upon Tyne NE4 6BN,

UK

1 Nava S, Confalonieri M, Rampulla C. Intermediate respiratory intensive care units in Europe: a European perspective. Thorax 1998;53:798-802.

2 Edbrooke DL, Stevens VG, Hibbert CL, et al. High dependency units in England: the lack of provision and the cost of addressing the shortfall. Care of the Critically Ill 1997;13:216-9.

3 Report of a Joint MRC/DH Working Party on Intensive and High Dependency Care Research. London, 1997.

ency Care Research. London, 1997.
4 Ryan DW, Bayly PJ, Weldon OG, et al. A propsective two month audit of the Ryan DW, Bayly PJ, Weldon OG, et al. A propsective two month audit of the
lack of provision of a high-dependency unit and its impact on intensive lack of provision of a high-depen
care. Anaesthesia 1997;52:265-70.

5 Bodenham AR, Klein H. High dependency units: role and need. Br f Hosp Med 1996;56:192-3.

6 Leeson-Payne CG, Aitkenhead AR. A prospective study to assess the demand for a high dependency unit. Anaesthesia 1995;50:383-7.

7 Singer M, Myers S, Hall G, et al. The cost of intensive care: a comparison on one unit between 1988 and 1991. Intensive Care Med 1994;20:542-9.

8 Bott J, Carroll MP, Conway JH, et al. Randomised controlled trial of nasal ventilation in acute ventilatory failure due to chronic obstructive airways disease. Lancet 1993;341:1555-7.

9 Kramer N, Meyer TJ, Meharg J, et al. Randomized, prospective trial of noninvasive positive pressure ventilation in acute respiratory failure. $\mathrm{Am} \mathcal{F}$ Respir Crit Care Med 1995;151:1799-806.

10 Brochard L, Mancebo J, Wysocki M, et al. Noninvasive ventilation for acute exacerbations of chronic obstructive pulmonay disease. $N$ Engl $f \mathrm{Med}$ exacerbations of

11 Chevrolet JC, Jolliet P, Abajo B, et al. Nasal positive pressure ventilation in patients with acute respiratory failure. Chest 1991;100:775-82.

12 Barbe F, Togores B, Rubi M, et al. Noninvasive ventilatory support does not facilitate recovery from acute respiratory failure in chronic obstructive pulmonary disease. Eur Respir f 1996;9:1240-5.

13 Department of Health. Guidelines on admission to and discharge from intensive care and high dependency care units. London, 1996. 